Reprod. Nutr. Dévelop., 1988, 28 (1), 125-126.

\title{
Dégradation microbienne dans le rumen du maïs normal et du maïs " brown mibrid " observée au microscope électronique à balayage
}

\author{
Elisabeth GRENET, P. BARRY
}

Laboratoire des Aliments,

I.N.R.A., Theix, 63122 Ceyrat, France.

Summary. Rumen microbial digestion of maize and of a less lignified brown midrib mutant has been observed by light microscope and scanning electron microscope.

La digestibilité du maïs (Zea Mays L.) " brown midrib bm 3 " (bm) est supérieure à celle du maïs normal (N) (Müller et al., 1972) et cette différence est attribuée à sa teneur plus faible en lignine $(5,7$ et $1,3 \%$ de la matière sèche respectivement pour $\mathrm{N}$ et bm selon la méthode de Van Soest) laquelle est de structure différente (Kuc et Nelson, 1964). Nous avons donc étudié à I'aide du microscope photonique et du microscope électronique à balayage (MEB) la structure histologique des tiges et des limbes du maïs $N$ et du maïs bm, mis en évidence la présence de lignine à l'aide de réactions histologiques spécifiques et observé la dégradation des tissus dans le rumen afin de mesurer l'influence du gène bm sur la qualité du maïs.

Matériel et méthodes. Six pieds de maïs $\mathrm{N}$ et six pieds de maïs bm (bm 3), provenant de la Station d'Amélioration des Plantes (I.N.R.A. Lusignan), ont été cultivés en serre (température $18^{\circ} \mathrm{C}$, éclairement $16 \mathrm{~h}$ par jour) et récoltés au stade pâteux - vitreux du grain. Dix fragments de $0,5 \mathrm{~cm}$ environ de haut ont été prélevés à mi-hauteur de l'entre-nœud situé sous l'épi femelle ainsi que dans le limbe correspondant et introduits dans des sachets de nylon. Ces sachets ont été placés avant la distribution du repas pendant $4 \mathrm{~h}, 8 \mathrm{~h}, 24 \mathrm{~h}, 48 \mathrm{~h}$ et $72 \mathrm{~h}$ dans le rumen d'une vache fistulisée alimentée avec de l'ensilage de maïs. Après leur sortie du rumen les échantillons ont été pour moitié fixés dans la glutaraldéhyde et séchés au point critique avant observation au MEB et pour l'autre moitié inclus dans la paraffine : des coupes de $16 \mu$ d'épaisseur ont été réalisées et colorées à la phloroglucine chlorhydrique ainsi qu'au réactif de Maüle.

Résultats et discussion. La dégradation microbienne débute très rapidement dans le mésophylle de la feuille, le parenchyme de la tige et le phloème. Seuls le xylème, les fibres des faisceaux libéro-ligneux, l'épiderme et le sclérenchyme sous-jacent résistent (fig. 1 et 2). La dégradation du parenchyme de la tige qui permet aux faisceaux libéro-ligneux de faire saillie à la surface de l'échantillon est plus rapide chez le bm que chez le $\mathrm{N}$ (fig. 3 et 4). Les parois du sclérenchyme de la tige sont moins épaisses chez le bm que chez le $\mathrm{N}$ et moins résistantes à la dégradation microbienne (fig. 5 et 6 ).

Les résultats obtenus montrent que le maïs bm est dégradé plus rapidement et plus complètement que le maïs $\mathrm{N}$ dans le rumen, ce qui peut expliquer l'amélioration des performances observées chez les animaux recevant les ensilages de maïs bm (Malterre et al., 1985).

Remerciements. - Ce travail a été réalisé en partie grâce à un financement accordé dans le cadre du GIS sur I'« Etude de la cellulolyse dans le rumen et en digesteur ". 

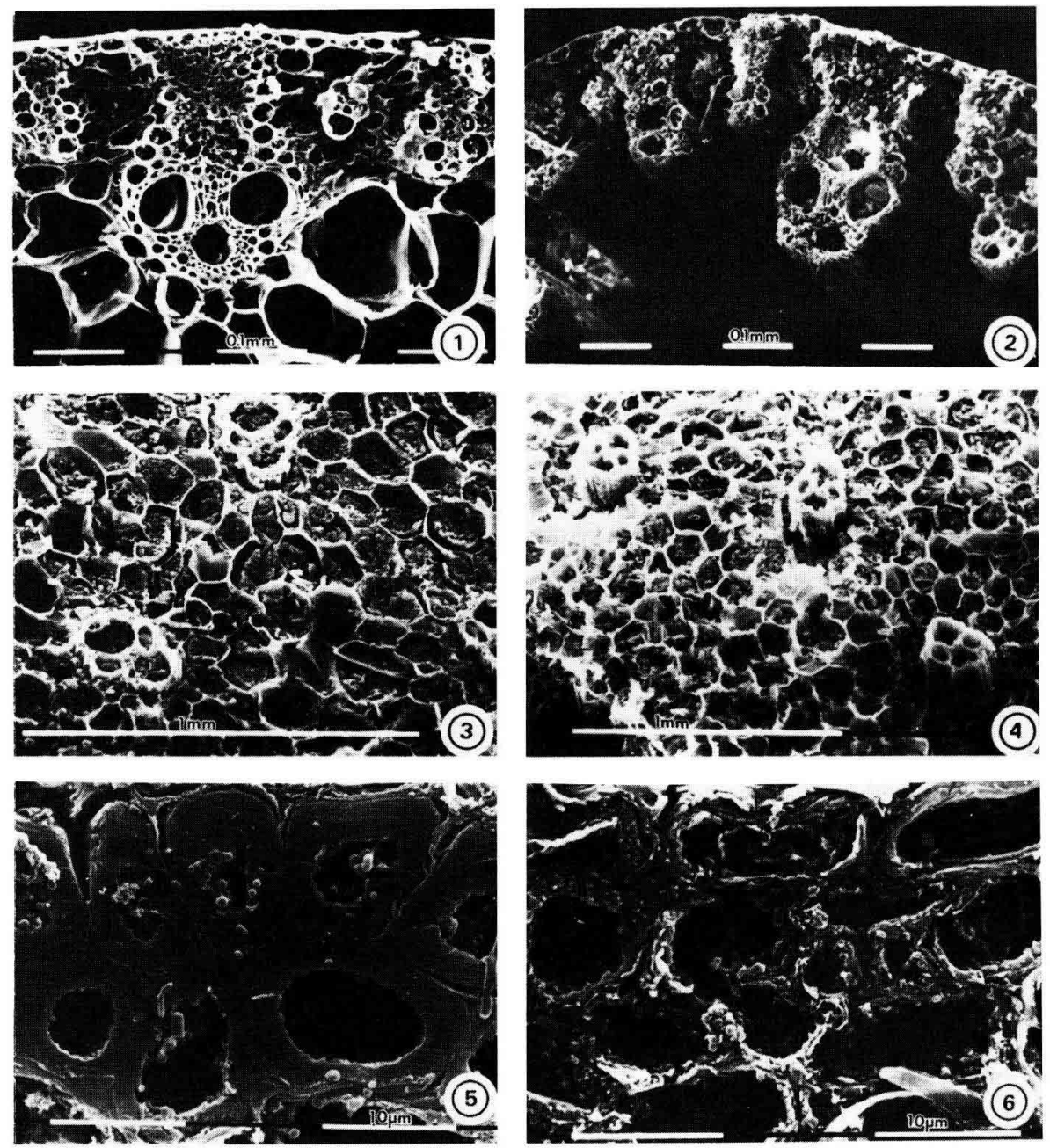

FIG. 1. - Coupe transversale d'un limbe de maïs $N$ témoin.

FIG. 2. - Coupe transversale d'un limbe de maïs $N$ ayant séjourné 72 h dans le rumen. Seuls les faisceaux libéro-ligneux, le sclérenchyme, colonisé par les champignons du rumen, et l'épiderme ont résisté à la dégradation.

FIG. 3. - Coupe transversale d'une tige de maïs $N$ ayant séjourné 24 h dans le rumen. Les faisceaux libéro-ligneux font légèrement saillie à la surface du parenchyme en cours de dégradation.

FIG. 4. - Coupe transversale d'une tige de maïs bm ayant séjourné 24 h dans le rumen. La digestion plus rapide du parenchyme pour ce maïs bm que pour le maïs $\mathrm{N}$ fait que les faisceaux libéro-ligneux font fortement saillie à la surface.

FlG. 5. - Coupe transversale du sclérenchyme de la tige de maïs $N$ ayant séjourné $72 \mathrm{~h}$ dans le rumen. Les parois lignifiées sont très épaisses.

FIG. 6. - Coupe transversale du sclérenchyme dans la tige de maïs bm avant séjourné $72 \mathrm{~h}$ dans le rumen. Les parois sont plus fines que celles du $\mathrm{N}$ et partiellement dégradées.

Kuc J., Nelson O. E., 1964. Arch. Biochem. Biophys., 105, 103-113.

Malterre C., Bertin G., Gallais A., Huguet L., Micol D., 1985. Bull. Tech. CRZV Theix I.N.R.A., $60,43-58$.

Müller L. D., Lechtenberg V. L., Bauman L. F., Barnes R. F., Rhykerd C. L., 1972. J. anim. Sci, 35, 883-889. 